\section{Rationale and protocol of MetNET-2 trial: Lanreotide Autogel plus metformin in advanced gastrointestinal or lung neuroendocrine tumors}

\author{
Sara Pusceddu ${ }^{* \neq, 1}$, Natalie Prinzi ${ }^{\neq, 1}$, Giuseppe Lo Russo', Daniela Femia', Massimo \\ Milione ${ }^{2}$, Federica Perrone ${ }^{3}$, Elena Tamborini ${ }^{4}$, Laura Concas ${ }^{1}$, Iolanda Pulice', \\ Claudio Vernieri', Francesca Corti', Roberto Buzzoni' \& Filippo de Braud ${ }^{1}$
}

\begin{abstract}
Metformin (MET) has recently emerged as a potentially active agent in cancer prevention and treatment. MET is thought to exert its antitumor effects either via modification of systemic metabolism or through cell-autonomous effects (e.g., activation of AMPK and inhibition of the mTOR pathway). Preliminary findings of the PRIME-NET study suggest that the addition of MET to treatment with everolimus (EVE) and/or somatostatin analogs (SSAs) can provide clinical benefit in diabetic neuroendocrine tumor (NET) patients. In light of this and other retrospective evidence of MET's anticancer activity in NETs, prospective studies are needed. A pilot, single-arm, open-label, prospective study (MetNET-2 trial, NCT02823691) was designed to evaluate the safety of MET in combination with lanreotide in well-differentiated gastrointestinal (WD GI) and lung NETs.
\end{abstract}

First draft submitted: 20 March 2017; Accepted for publication: 11 May 2017; Published online: 5 June 2017

Neuroendocrine tumors (NETs) are a group of malignancies that arise from neuroendocrine cells residing in all the tissues of the body and that can dedifferentiate into tumor cells. Once considered rare, the incidence of NETs has risen worldwide in recent years [1,2]. The Surveillance, Epidemiology and End Results Registry identified 64,971 patients with NETs in the USA in the period from 1973 to 2012, with a significant increase in the reported annual age-adjusted incidence rate of NETs from $1.09 / 100,000$ to $6.98 / 100,000$ [1].

Somatostatin analog (SSA) therapy with Lanreotide Autogel ${ }^{\circledR}$ (Ipsen, Slough, UK [LAN, administered every 28 days at the maximum dose of $120 \mathrm{mg}$ ]) or octreotide long-acting release (OCT LAR) constitutes one of the most important therapeutic options for the gastrointestinal (GI) and lung carcinoid treatment in both functioning and nonfunctioning tumors. These long-acting SSAs are considered the gold standard therapy for the control of endocrine syndromes associated with NETs. In GI and pancreatic (p) NETs, complete or partial symptom control is generally

'Department of Medical Oncology, Unit 1, ENET Center of Excellence, Fondazione IRCCS Istituto Nazionale Tumori, Via G. Venezian 1, 20133 Milan, Italy

2Department of Pathology, ENET Center of Excellence, Fondazione IRCCS Istituto Nazionale Tumori, Via G. Venezian 1, 20133 Milan, Italy ${ }^{3}$ Department of Pathology, Laboratory of Experimental Molecular Pathology, ENET Center of Excellence, Fondazione IRCCS Istituto Nazionale Tumori, Via G. Venezian 1, 20133 Milan, Italy

${ }^{4}$ Department of of Diagnostic Pathology \& Laboratory Medicine ENET Center of Excellence, Fondazione IRCCS Istituto Nazionale

Tumori, Via G. Venezian 1, 20133 Milan, Italy

*Author for correspondence: Tel.: +39 02 23902500; Fax: +39 02 23902149; sara.pusceddu@istitutotumori.mi.it

₹Authors contributed equally

\section{KEYWORDS}

- lanreotide • metformin

- well-differentiated NETs

Future : Medicine ${ }_{\text {part of }}$ 
achieved in at least $50 \%$ of syndromic patients receiving SSA therapy, though clinical response in over $70-90 \%$ of functioning NETs has been reported [3-5].

Two randomized, placebo-controlled, Phase III trials (PROMID and CLARINET studies) demonstrated that OCT and LAN can exert an antiproliferative effect in patients with advanced gastroenteropancreatic (GEP)NETs [6,7]. Prospective data on the antiproliferative effect of SSAs in well-differentiated (WD) bronchopulmonary NETs are lacking, but limited information from retrospective, case-list and unrandomized studies shows a significant percentage of disease stabilization in the majority of patients analyzed [8,9].

New combinations with SSAs and other investigational drugs are currently under evaluation with the aim to improve clinical outcomes, while maintaining a good tolerability profile and health-related quality of life. MetNET-2 is one of the first studies designed to explore the safety of Metformin (MET) in combination with LAN in advanced GI NETs and lung carcinoids.

\section{Study rationale}

SSAs have a direct and an indirect antiproliferative effect on neuroendocrine neoplasms [10,11]. SSAs indirectly induce the inhibition of growth factor secretion (GH, IGF-1, FGF, VEGF, EGF, PDGF, TGF) and tumor angiogenesis by interacting with peritumoral vascular somatostatin-2 (SST-2) receptors and inhibiting growth factors. Immunomodulatory effects are mediated by natural killer lymphocytes and monocytes [10]. SST receptors 1-5 directly inhibit cell proliferation by downregulation of $M A P K$ pathways and SST2/3 induce apoptosis by activation of the $p 53 / B A X$ pathway [10,11]. The primary or secondary mechanism of SSA resistance is usually associated with the compensatory overexpression of intracellular drivers activated by growth factors targeting IGF-1, VEGF and PDGF receptors and through the dysregulation and constitutive activation of the PI KK/AKT/mTOR pathway and genetic aberration (mutation of phosphate and tensin homolog, PI3K, TSC2, NF1 and LKB1) [10].

Evidence for the antiproliferative effect of LAN $120 \mathrm{mg}$ is supported by clinical trial efficacy data on progression-free survival (PFS) in patients with GEP NETs published in July 2014 (CLARINET study) [7]. The study showed that in 204 patients with nonfunctioning GEP
NETs, naïve to previous SSA treatment, there was a $53 \%$ risk reduction of death or disease progression after 24 months of treatment with LAN $120 \mathrm{mg}$ every 28 days compared with placebo (HR: 0.47; 95\% CI: 0.30-0.73; p < 0.001).

A number of studies have identified diabetic patients being at an increased risk for the development of cancer [12]. MET is a widely prescribed antidiabetic drug with established efficacy, coupled with a favorable safety profile and low cost. An increasing number of studies have associated MET treatment with a decrease in cancer risk. Moreover, MET has also been associated with improved outcomes in cancer patients [12-16]. In a recently published systematic review and metaanalysis, Coyle et al. [16] enrolled 24,178 patients affected by colorectal, prostate, breast and urothelial cancer. The authors showed that MET use was associated with a significant benefit in all the outcomes, in particular in early-stage colorectal cancer and prostate cancer, suggesting its use as an adjuvant agent [16]. These possible pleiotropic effects of MET may establish it as a cancer prevention and treatment option. Larger studies are expected to better investigate the potential antineoplastic effects of MET. The possible molecular antiproliferative effect of MET is characterized by modulation of insulin secretion and IGF-1R (indirect mechanism) and by activation of $A M P K$ mediated by $L K B 1$ oncogene expression (direct mechanism). This driver is also correlated with the negative modulation of the Ras/ Raf/MAPK pathway and the TSC1-2/mTOR complex $[17,18]$. The anticancer effects of MET on human pancreatic neuroendocrine BON 1 cells in vitro are known. MET dose dependently inhibited neuroendocrine BON 1 cell viability, with suppression of $m$ TORC1 signaling evidenced by the suppression of 4ebp1, pP70S6K and $\mathrm{S} 6$ phosphorylation, with compensatory AKT activity [19].

Available data about the effects on NETs of SSAs and MET in association are limited. The most relevant and recent clinical data include a retrospective analysis by the Spanish neuroendocrine study group [20], and the PRIMENET study, designed after evidence from a small mono-institutional retrospective cohort of patients that MET intake was associated with increased PFS in diabetic pNET patients receiving everolimus (EVE) plus OCT [21,22]. In the Spanish study, 92 patients with advanced G1-G2 GEP and lung NETs received EVE: 25 were diabetics and 11 were treated with MET. A better 
outcome was achieved in the patients treated with MET than in the diabetic patients treated with non-MET agents (median PFS: 34.1 vs 8.2 months, respectively, HR: $0.12 ; 95 \% \mathrm{CI}$ : 0.02-0.57; $\mathrm{p}=0.008$ ) [20]. The retrospective PRIME-NET study evaluated 445 patients (209 normoglycemic and 236 hyperglycemic) with advanced WD pNET treated with EVE with or without SSAs at 24 Italian centers. Overall, median PFS was 15.1 months in the normoglycemic patients versus 32 months in the hyperglycemic groups (HR: 0.63; 95\% CI: $0.50-0.80 ; \mathrm{p}=0.0002)$ [22].

In particular, the study highlighted a statistically significant difference in the median PFS among diabetic patients receiving MET (44.2 months) versus diabetic patients receiving insulin or diet (20.8 months) and normoglycemic patients (15.1 months), respectively (HR: 0.45 ; 95\% CI: $0.32-0.62$; $p<0.00001$ for comparison between patients taking MET and nornoglycemic patients) [22]. Several factors may potentially bias these experiences. Firstly, all are retrospective studies. Moreover, the potential role of important factors such as surgery, dosage and duration of exposure to MET are not always clearly specified. Finally, the selected inclusion criteria are often too vague to identify the real effect of MET. Prospective evaluation is needed to confirm these findings. The Fondazione IRCCS Istituto Nazionale dei Tumori, Milan, is currently conducting a prospective, singlecenter, open-label, Phase II, proof-of-concept study (MetNET1 trial, NCT02294006) to investigate the activity and safety of EVE in combination with OCT and MET in patients with advanced WD pNETs [23]. Moreover, building on these preliminary data, we designed the MetNET-2 trial. The aim of this second trial is to define the safety of concomitant treatment with LAN and MET in progressive advanced GI NETs or lung carcinoids, and to evaluate as a secondary objective the possible synergistic effect of this combination.

\section{Treatment plan}

\section{- Study design \& target population}

MetNET-2 is a pilot, single-arm, open-label, prospective study designed to evaluate the safety of LAN (120 mg every 28 days) in combination with MET (2550 mg daily) in patients with advanced progressive WD GI NETs or lung carcinoids. The study population will include 20 normoglycemic and/or hyperglycemic patients with a histologically confirmed diagnosis of WD G1-G2 NET [24,25]. Patients will continue therapy until radiological disease progression or intolerable toxicity. The planned enrollment period is 24 months and the overall study duration is about 3 years.

\section{- Primary \& secondary objectives}

The primary objective is the evaluation of the safety profile, as assessed by the incidence report of adverse events (AEs) and severe AEs (SAEs). An AE is defined as any undesirable medical condition or significant change in laboratory parameters that occurs at any time from the informed consent signature until the end of the study, even if no study drug has been administered. An SAE is defined as any AE that results in death, that is life threatening, that places the subject at immediate risk of death or that results in hospitalization, prolongation of existing hospitalization or in a persistent significant disability. AE intensity will be determined using the latest version of the National Cancer Institute Common Terminology Criteria for AEs ( CTCAE Scale ver. 4.03).

Evaluation of the activity of LAN in combination with MET is a secondary exploratory objective. According to Response Evaluation Criteria in Solid Tumors (RECIST) version 1.1 [26] the objective response rate and disease control rate will be evaluated. Furthermore, time to progression, defined as the time from the first study drugs administration to the first clinical, biochemical or radiological progression will be assessed. Another secondary objective is the evaluation of the prognostic and predictive role of genetic biomarkers in treated patients. We plan to investigate several genes involved in the pathways related to MET and/or SSA activity. Tumor samples will be analyzed using a custom panel covering the coding sequences of 111 genes, including $L K B 1$, TP53, KRAS, TP53/BAX, IGF1R, VEGFR, PDGFR and $A K T$, PIK3CA, phosphate and tensin homolog, $m T O R, T S C 2$ and NF1.

\section{- Dose \& schedule of therapy}

Patients will receive LAN (120 mg every 28 days, equivalent to one cycle), plus MET (2550 mg daily maximum dose, as oral administration). If well tolerated, the MET starting dose of $850 \mathrm{mg} /$ day will be increased up to $1700 \mathrm{mg} /$ day at day 14 and up to $2550 \mathrm{mg} /$ day at day 28 (maximum dose). Clinical and physical examination, tolerability assessment and laboratory 


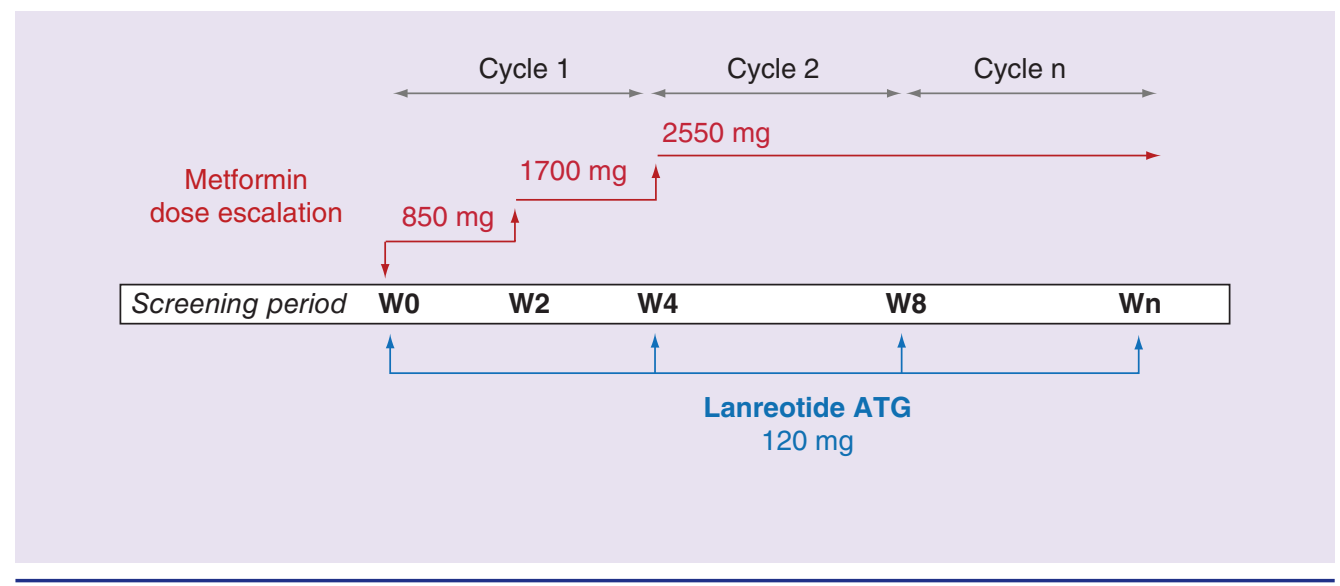

Figure 1. MetNET-2 trial: study design and schedule. The treatment will be administered until disease progression or intolerable toxicity. The metformin starting dose of $850 \mathrm{mg} / \mathrm{day}$, will be increased up to $1700 \mathrm{mg} /$ day at day 14 and up to $2550 \mathrm{mg} /$ day at day 28 (maximum dose), if well tolerated. Lanreotide Autogel $120 \mathrm{mg}$ will be administered every 28 days.

$\mathrm{W}=$ week

evaluations (renal function, liver function, metabolic assessment, venous blood gas, urinalysis, C-peptide and insulin) will be performed at days 7, 14 and 28 (and at further visits) to evaluate the safety of the combination therapy until the maximum MET dose. In patients intolerant to the escalating or the maximum MET dose, the treatment dose will be reduced and tolerability retested. The treatment period is scheduled to be until clinical or radiological disease progression, according to RECIST version 1.1 (Figure 1).

\section{- Statistical plan}

Sample size \& power consideration

A single-stage A'Hern design will be used for the sample size determination. The null hypothesis that the SAE rate (as measured on the CTCAE Scale ver. 4.03) related to treatment is $25 \%$ will be tested against a one-sided alternative. Twenty patients will be enrolled. The null hypothesis will be rejected if not more than 2 out of 20 patients experience severe toxicity. This design yields a type I error rate of $10 \%$ and a power of $85 \%$ with a maximum of toxicity rate of $5 \%$.

Demographic \& other baseline characteristics Descriptive statistics (means and standard deviation or frequency tables, depending on their nature) will be used to summarize demographic characteristics, medical history and physical examination abnormalities of all patients included in the study. Concomitant medications will be summarized by individual data listings and summary tables.

\section{Populations}

The following populations will be used in the analysis of this study: the Safety population will comprise all those patients who receive at least one dose of study drugs combination, as soon as any of the two drugs is received. The Intention-to-Treat population will comprise all those patients who receive at least one dose of study drugs combination and who have at least one efficacy evaluation after baseline visit.

\section{Analysis of feasibility}

Descriptive statistics are calculated for efficacy data from the efficacy population (Intention-toTreat population: all patients who received at least one dose of LAN plus MET). Descriptive rather than inferential statistics are performed because this is a noncomparative exploratory investigation.

Interim analysis

No interim analysis is foreseen for the study.

Statistical assumptions \& statistical software Statistical tables and analyses will be conducted using $S A S^{\circledR}$, version 9.2 or higher, statistical software package.

\section{- Ethics, informed consent \& safety}

The study protocol and procedures were designed following Good Clinical Practice guidelines and the principles set forth by the Helsinki Declaration. Written and signed informed consents will be obtained from all the enrolled patients before any study-specific procedure takes place. 
Table 1. Study procedures and assessments.

\begin{tabular}{|c|c|c|c|c|c|c|c|c|}
\hline Procedure and assessments & $\begin{array}{l}\text { Screening } \\
\text { period }\end{array}$ & $\begin{array}{l}\text { Wo (start } \\
\text { treatment) }\end{array}$ & W2 & W4 & W6 & W8 & $\begin{array}{l}\text { Wn } \\
\text { (every } \\
4 \text { weeks) }\end{array}$ & EOS \\
\hline Clinical history and demography & $x$ & & & & & & & \\
\hline Prior and concomitant medications & $\mathrm{x}$ & $\mathrm{x}$ & $x$ & $\mathrm{X}$ & $x$ & $x$ & $x$ & $\mathrm{x}$ \\
\hline $\begin{array}{l}\text { Clinical and physical examination, } \\
\text { bodyweight, assessment of blood } \\
\text { pressure, saturation test, vital signs }\end{array}$ & $\mathrm{x}$ & $x$ & $\mathrm{X}$ & $\mathrm{x}$ & $\mathrm{x}$ & $\mathrm{x}$ & $x$ & $\mathrm{X}$ \\
\hline Written informed consent & $x$ & & & & & & & \\
\hline $\begin{array}{l}\text { Clinical laboratory tests (renal function, } \\
\text { liver function, glucose, lipid profile, } \\
\text { venous blood gas, HbA1c level, urinalysis, } \\
\text { C-peptide, insulin) }\end{array}$ & $\mathrm{x}$ & $x$ & $x$ & $\mathrm{x}$ & $x$ & $x$ & $x$ & $\mathrm{X}$ \\
\hline HBV and HCV viral markers & $x$ & & & & & & & \\
\hline Oral glucose tolerance test & $x$ & & & & & & & \\
\hline Dosage of Cga, NSE and urinary 5-HIAA ${ }^{\neq}$ & $x$ & $x$ & & & & & $\mathrm{X}^{\dagger}$ & $\mathrm{X}$ \\
\hline Dosage of VitB12, FT3, FT4, TSH & $x$ & $x$ & & & & & $\mathrm{X}^{\dagger}$ & $x$ \\
\hline ECG, cardiac evaluation and $\mathrm{Echo}^{\S}$ & $\mathrm{X}$ & & & & & & $X^{\S}$ & $X^{\S}$ \\
\hline Likert scale evaluation collection ${ }^{\neq}$ & $\mathrm{X}$ & $x$ & $\mathrm{x}$ & $x$ & $x$ & $x$ & $\mathrm{X}$ & $x$ \\
\hline CT scan or MRI, with or without contrast & $x$ & & & & & & $\mathrm{X}^{\dagger}$ & \\
\hline Tumor sample collection & $x$ & & & & & & & \\
\hline Evaluation of increasing metformin dose & & & $x$ & $\mathrm{X}$ & $\mathrm{x}$ & & & \\
\hline Adverse events & & & $x$ & $\mathrm{X}$ & $\mathrm{x}$ & $x$ & $\mathrm{X}$ & $\mathrm{X}$ \\
\hline Serious adverse events & & & $\mathrm{X}$ & $\mathrm{X}$ & $\mathrm{X}$ & $\mathrm{X}$ & $\mathrm{X}$ & $\mathrm{X}$ \\
\hline \multicolumn{9}{|c|}{$\begin{array}{l}+5 \text { Requested every } 4 \text { months after starting therapy. } \\
+\neq 5 \text { Only in functioning patients. } \\
+\neq 5 \text { Only if clinically required. } \\
\text { 5-HIAA: } 5 \text {-Hydroxyindoleacetic acid; CgA: Chromogranin A; CT: Computed tomography; Echo: Echocardiogram; ECG: Electrocardiogram; EOS: End of study; NSE: Neuron-specifi } \\
\text { enolase; W: Week; HBV: Hepatitis B virus, HCV: Hepatitis C virus. }\end{array}$} \\
\hline
\end{tabular}

\section{- Criteria}

Inclusion criteria: adult patients (male or female, age older than 18 years) with advanced nonresectable, progressive NETs within 6 months before study inclusion; histological diagnosis of WD (G1 and G2) GI or lung NETs; Eastern Cooperative Oncology Group performance status (ECOG PS) $\leq 2$; life expectancy of more than 12 months; functioning or nonfunctioning NETs; type 2 diabetic or normoglycemic patients (the assessment of Oral Glucose Tolerance Test, according to American Diabetes Association guidelines 2015 [27], will be performed in screening phase); tumor tissue available for analysis; documented OctreoScan/positron emission tomography (PET) $\mathrm{Ga}^{68}$ uptake/ immunohistochemical stain of SST-2 receptors within 6 months before study entry; adequate liver, kidney and bone marrow function. Written informed consents will be obtained before any study-specific procedure takes place (Table 1).

\section{- Study limitations}

The most important limitations of this study are the small number of patients planned for this analysis, and the lack of a control group, which prohibits comparative analysis.

We remark that the primary objective of the MetNET-2 trial will be the evaluation of the safety and the tolerability of the combination of LAN with MET; therefore, evaluation of the efficacy (objective response rate, disease control rate and time to progression) of this regimen will be only an exploratory objective.

In this preliminary pilot evaluation, we will enroll patients with both WD GI NETs and lung carcinoids, despite differences in their clinical management and prognosis. This heterogeneity is a further limitation of the study, but this explorative analysis could be helpful for the selection of patients for any future prospective Phase II trial.

\section{Conclusion}

The aim of this pilot, single-arm, open-label, prospective study is to examine the safety of LAN in combination with MET in WD GI NETs or lung carcinoids. Although in a small 
and heterogeneous population, the MetNET-2 trial has the potential to generate useful preliminary data about the possible synergistic effects of this combination, with the rationale to evaluate this combination in a future Phase II trial in both diabetic and normoglycemic patients and in both WD GI and/or lung NET patients. Moreover, the evaluation of the prognostic and predictive role of genetic biomarkers in treated WD GI NETs or lung carcinoids could be a future area of focus in patient treatment selection.
Financial \& competing interests disclosure

$S$ Pusceddu received honoraria from Novartis, Ipsen, Italfarmaco, Pfizer. N Prinzi and D Femia received honoraria from Italfarmaco, $R$ Buzzoni received honoraria from Novartis, Ipsen, Italfarmaco, Pfizer. The authors have no other relevant affiliations or financial involvement with any organization or entity with a financial interest in or financial conflict with the subject matter or materials discussed in the manuscript apart from those disclosed.

Editorial assistance in the preparation of this manuscript was provided by Kenneth Adolf Britsch of Avicenna inc (funded by IPSEN).

\section{EXECUTIVE SUMMARY}

\section{Study rationale}

- The CLARINET study involving 204 patients with GI NETs showed a 53\% reduction in the risk of death/progression of disease versus placebo.

- SSAs have a direct and an indirect antiproliferative effect on neuroendocrine neoplasms.

- Preliminary findings of the PRIME-NET study suggest that the addition of MET to therapy with EVE and/or SSAs alone can provide clinical benefit in diabetic NET patients.

\section{Study design}

- MetNET-2 is a pilot, single-arm, open-label, prospective study with the aim to evaluate the safety of Lanreotide Autogel (LAN; 120 mg every 28 days) in combination with MET ( 2550 mg daily) in 20 normoglycemic and/or hyperglycemic patients with advanced, progressive, WD GI NETs or lung carcinoids.

\section{Primary \& secondary objectives}

- The primary objective is the evaluation of the safety profile, as assessed by the incidence report of AEs and SAEs.

- The secondary objective: evaluation of the activity of LAN in combination with MET and the evaluation of the prognostic and predictive role of genetic biomarkers in treated patients.

\section{Dose \& schedule of therapy}

- Patients will receive LAN (120 mg every 28 days, equivalent to one cycle), plus MET ( $2550 \mathrm{mg}$ daily maximum dose, as oral administration). If well tolerated, the MET starting dose of $850 \mathrm{mg} /$ day will be increased up to $1700 \mathrm{mg} /$ day at day 14 and up to $2550 \mathrm{mg} /$ day at day 28 (maximum dose).

- The treatment period is scheduled to be until clinical or radiological disease progression, according to RECIST version 1.1.

\section{Criteria}

- Inclusion criteria: adult patients with advanced nonresectable, progressive NETs; histological diagnosis of WD (G1 and G2) Gl or lung NETs; ECOG PS $\leq 2$; life expectancy of more than 12 months; functioning or nonfunctioning NETs; type 2 diabetic or normoglycemic patients; tumor tissue available for analysis; documented OctreoScan/PETGa ${ }^{68}$ uptake/ immunohistochemical stain of SST-2 receptors within 6 months before study entry; adequate liver, kidney and bone marrow function. Written informed consent will be obtained before any study-specific procedure takes place.

\section{References}

Papers of special note have been highlighted as:

- of interest; $\bullet$ of considerable interest

1 Dasari A, Shen C, Halperin D et al. Trends in the incidence, prevalence, and survival outcomes in patients with neuroendocrine tumors in the United States. JAMA Oncol. doi:10.1001/jamaoncol.2017.0589 (2017) (Epub ahead of print).

2 Hallet J, Law CH, Cukier M, Saskin R, Liu $N$, Singh S. Exploring the rising incidence of neuroendocrine tumors: a population-based analysis of epidemiology, metastatic presentation, and outcomes. Cancer 121(4), 589-597 (2015).

3 Modlin IM, Pavel M, Kidd M, Gustafsson BI. Review article: somatostatin analogues in the treatment of gastroenteropancreatic 
neuroendocrine (carcinoid) tumours. Aliment. Pharmacol. Ther. 31(2), 169-188 (2010).

4 Wolin EM. The expanding role of somatostatin analogs in the management of neuroendocrine tumors. Gastrointest. Cancer Res. 5(5), 161-168 (2012).

5 Culler MD, Oberg K, Arnold R, Krenning EP, Sevilla I, Díaz JA. Somatostatin analogs for the treatment of neuroendocrine tumors. Cancer Metastasis Rev. 30(1), 9-17 (2011).

6 Rinke A, Müller HH, Schade-Brittinger C et al. Placebo-controlled, double-blind, prospective, randomized study on the effect of octreotide LAR in the control of tumor growth in patients with metastatic neuroendocrine midgut tumors: a report from the PROMID study group. J. Clin. Oncol. 27(28), 4656-4663 (2009).

- Phase III study that demonstrated the efficacy of octreotide long-acting release in patients with metastatic neuroendocrine midgut tumors.

7 Caplin ME, Pavel M, Ruszniewski P et al. Lanreotide in metastatic enteropancreatic neuroendocrine tumors. N. Engl. J. Med. 371(16), 1556-1557 (2014).

- Phase III study that demonstrated the antiproliferative effect of lanreotide in patients with nonfunctioning gastroenteropancreatic neuroendocrine tumors.

8 Filosso PL, Ruffini E, Oliaro A, Papalia E, Donati G, Rena O. Long-term survival of atypical bronchial carcinoids with liver metastases, treated with octreotide. Eur. J. Cardiothorac. Surg. 21(5), 913-917 (2002).

9 Öberg K, Hellman P, Ferolla P, Papotti M, ESMO Guidelines Working Group. , Neuroendocrine bronchial and thymic tumors: ESMO Clinical Practice Guidelines for diagnosis, treatment and follow-up. Ann. Oncol. 23(7), 120-123 (2012).

10 Susini C, Buscail L. Rationale for the use of somatostatin analogs as antitumor agents. Ann. Oncol. 17(12), 1733-1742 (2006).

11 Sharma K, Patel YC, Srikant CB. Subtypeselective induction of wild-type $\mathrm{p} 53$ and apoptosis, but not cell cycle arrest, by human somatostatin receptor 3. Mol.

Endocrinol. 10(12), 1688-1696 (1996).

12 Klil-Drori AJ, Azoulay L, Pollak MN. Cancer, obesity, diabetes, and antidiabetic drugs: is the fog clearing? Nat. Rev. Clin. Oncol. 14(2), 85-99 (2017).

13 Rizos CV, Elisaf MS. Metformin and cancer. Eur. J. Pharmacol. 705(1-3), 96-108 (2013).

14 Kasznicki J, Sliwinska A, Drzewoski J. Metformin in cancer prevention and therapy. Ann. Transl. Med. 2(6), 57 (2014).

15 Decensi A, Puntoni M, Goodwin P et al. Metformin and cancer risk in diabetic patients: a systematic review and metaanalysis. Cancer Prev. Res. (Phila). 3(11), 1451-1461 (2010).

16 Coyle C, Cafferty FH, Vale C, Langley RE. Metformin as an adjuvant treatment for cancer: a systematic review and meta-analysis. Ann. Oncol. 27(12), 2184-2195 (2016).

- Meta-analysis suggesting that metformin could be a useful adjuvant agent in colorectal and prostate cancer.

17 Towler MC, Hardie DG. AMP-activated protein kinase in metabolic control and insulin signaling. Circ. Res. 100(3), 328-341 (2007).

18 Vázquez-Martín A, Oliveras-Ferraros $\mathrm{C}$, del Barco S, Martín-Castillo B, Menéndez JA. mTOR inhibitors and the antidiabetic biguanide metformin: new insights into the molecular management of breast cancer resistance to the HER2 tyrosine kinase inhibitor lapatinib (Tykerb). Clin. Transl. Oncol. 11(7), 455-459 (2009).

19 Vlotides G, Tanyeri A, Spampatti M et al. Anticancer effects of metformin on neuroendocrine tumor cells in vitro. Hormones (Athens) 13(4), 498-508 (2014).

20 Custodio $\mathrm{A}$, Jimènez-Fonseca $\mathrm{P}$, AlonsoOrudna V et al. Prognostic role od diabetes mellitus (DM) and Metformin therapy in patients (pts) with advanced G1-G2 neuroendocrine tumors (NETs) treated with Everolimus (EVE). Presented at: 12th ENETS Congress. Barcelona, Spain, 11-13 March 2015.

- Retrospective study that showed a better outcome in diabetic patients with advanced G1-G2 gastroenteropancreatic and lung neuroendocrine tumors, treated with everolimus and metformin compared with diabetic patients treated with everolimus and non metformin agents.

21 Pusceddu S, Buzzoni R, Vernieri C et al. Metformin with everolimus and octreotide in pancreatic neuroendocrine tumor patients with diabetes. Future Oncol. 12(10), 1251-1260 (2016).

22 Pusceddu S, Marconcini R, Spada F et al. Metformin impact on progression-free survival in diabetic patients with advanced pancreatic neuroendocrine tumors ( $\mathrm{pNET}$ ) receiving everolimus and/or somatostatin analogues. The PRIME-NET (Pancreatic multicentric, Retrospective, Italian MEtformin) study. Presented at: ESMO Congress. Copenhagen, Denmark, 7-11 October 2016.

-• Metformin is associated with improved progression-free survival in patients with advanced pneuroendocrine tumors, and could be effective in combination with both somatostatin analogs and everolimus.

23 Pusceddu S, de Braud F, Concas L et al. Rationale and protocol of the MetNET-1 trial, a prospective, single center, Phase II study to evaluate the activity and safety of everolimus in combination with octreotide LAR and metformin in patients with advanced pancreatic neuroendocrine tumors. Tumori. 100 (6), e286-e289 (2014).

- Phase II study that explores the antiproliferactive effect of metformin in combination with targeted agents (everolimus and octreotide long-acting release) in advanced pancreatic welldifferentiated neuroendocrine tumor.

24 Bosman FT, Carneiro F, Hruban RH, Theise ND. WHO Classification of Tumours of the Digestive System (4th Edition). IARC Press, Lyon, France (2010).

25 Travis WD, Brambilla E, Burke AP, Marx A, Nicholson AG. WHO Classification of Tumours of the Lung, Pleura, Thymus and Heart (4th Edition). IARC Press, Lyon, France (2015).

26 Eisenhauera EA, Therasseb P, Bogaertsc J et al. New response evaluation criteria in solid tumours: revised RECIST guideline (version 1.1). Eur. J. Cancer 45(2), 228-247 (2009).

27 Garber AJ, Abrahamson MJ, Barzilay JI et al. Consensus statement by the American Association of clinical endocrinologist and American college of endocrinology on the comprehensive tipe-2 diabetes management algorithm - 2015 Executive Summary. Endocr. Pract. 21(12), 1403-1414 (2015). 